\section{Tratamiento con vemurafenib: indicación de mapeo corporal y seguimiento digital dermatoscópico}

Camila Otero ${ }^{1}$, Daniela Capurro ${ }^{1}$, Sofia Sellanes ${ }^{1}$, Andrea Nicola $^{2}$, Lídice Dufrechou ${ }^{3}$, Sofía Nicoletti ${ }^{4}$, Alejandra Larre Borges ${ }^{5}$

\section{RESUMEN}

El Vemurafenib es un inhibidor de la enzima serina/ treonina quinasa BRAF utilizado en el tratamiento de pacientes con melanoma con diseminación locorregional y enfermedad metastásica, portadores de la mutación V600E del gen BRAF. Se ha asociado a múltiples efectos adversos cutáneos de los cuales se destaca la posibilidad de generar cambios en los nevos melanocíticos, aparición de nuevos nevos e incluso de segundos melanomas. El seguimiento digital dermatoscópico con mapeo corporal, ha demostrado utilidad en el diagnóstico precoz de melanoma.

Presentamos dos casos clínicos de pacientes con antecedentes de melanoma en tratamiento con inhibidores de $\mathrm{BRAF}$ (BRAFi) e inhibidores de BRAF y MEK (MEKi) en quienes se realizó seguimiento digital dermatoscópico con mapeo corporal. Se detectaron cambios en nevos melanocíticos preexistentes, aparición segundos melanomas y metástasis cutáneas.

El grupo de pacientes con antecedentes de melanoma y en tratamiento con BRAFi o combinación de BRAFi y MEKi se beneficia especialmente del control dermatológico con seguimiento digital dermatoscópico y mapeo corporal.

Palabras clave: Dermatoscopía; melanoma; seguimiento digital; mapeo corporal

\section{SUMMARY}

Vemurafenib is an inhibitor of the serine / threonine kinase BRAF enzyme currently used in the treatment of patients with locoregional spread and metastatic melanoma carriers of the mutationV600E of the BRAF gene. It has been associated with multiple cutaneous adverse effects including changes in melanocytic nevi, appearance of new nevi and even second melanomas. Dermoscopic digital follow-up with total body mapping has proven useful in the early diagnosis of melanoma.

We present two cases of patients with a history of melanoma in treatment with BRAF inhibitors (BRAFi) and inhibitors of BRAF and MEK (MEKi) in whom a digital dermoscopic follow-up was performed with body mapping. Changes in preexisting melanocytic nevi, second melanomas and cutaneous metastases were detected.

The group of patients with a history of melanoma and in treatment with BRAFi or a combination of BRAFi and MEKi especially benefits from dermatological surveillance with digital dermoscopic followup and total body mapping.

Key words: Dermoscopy; melanoma; digital followup; body mapping
$\mathrm{E}$ 1 Vemurafenib es un inhibidor de la enzima serina/treonina quinasa B-RAF utilizado en el tratamiento de pacientes con melanoma con diseminación locorregional y enfermedad metastásica, portadores de la mutación V600E del gen BRAF. Desde su implementación como protocolo en el tratamiento del melanoma se ha asociado a múltiples efectos adversos cutáneos de los cuales se destaca la posibilidad de generar cambios en los nevos melanocíticos, aparición de nuevos nevos ${ }^{1,2,3}$ e incluso de segundos melanomas ${ }^{4,5,6}$. Otros efectos adversos cutáneos reportados son: prurito, rash, fotosensibilidad, alopecia, xerosis, carcinoma espinocelular, queratoacantoma y eritrodisestesia palmo-plantar. A pesar de estos efectos adversos, en raras oportunidades se discontinúa el tratamiento con vemurafenib. $^{7}$

La dermatoscopía es una técnica in vivo no invasiva que permite la magnificación de las lesiones mediante el uso de una lente llamada dermatoscopio. A través de la luz polarizada se logra borrar las capas superficiales de la piel con una mejor visualización de estructuras más
${ }^{1}$ Residente Cátedra de Dermatología, Hospital de Clínicas "Dr. Manuel Quintela", MontevideoUruguay. ${ }^{2}$ Asistente Cátedra de Dermatología, Hospital de Clínicas "Dr. Manuel Quintela", Montevideo-

Uruguay.

${ }^{3}$ Profesora Adjunta Interina Cátedra de Dermatología,

Hospital de

Clínicas "Dr.

Manuel Quintela",

Montevideo-

Uruguay.

${ }^{4}$ Profesora Adjunta

Cátedra de

Dermatología,

Hospital de

Clínicas "Dr.

Manuel Quintela",

Montevideo-

Uruguay.

${ }^{5} \mathrm{Ex}$ Profesora

Adjunta Cátedra

de Dermatología,

Hospital de

Clínicas " Dr.

Manuel Quintela",

Montevideo-

Uruguay.

Trabajo no recibió financiamiento.

Los autores declaran no tener conflictos de interés.

Recibido el 20 de mayo de 2019, aceptado el 28 de junio de 2019.

Correspondencia a: Camila Otero

E-Mail: oterolarreborges@ gmail.com 
profundas lo cual mejora la precisión del diagnóstico de tumores cutáneos, principalmente de melanoma. ${ }^{8}$ Su uso, en comparación con la inspección a ojo desnudo, ha aumentado significativamente la precisión diagnóstica de melanoma entre un 5 y un $30 \%$ entre los observadores entrenados. ${ }^{9}$

El seguimiento digital dermatoscópico con mapeo corporal (SDDMG) consiste en el registro de imágenes de zonas corporales y registro dermatoscópico de las lesiones pigmentadas presentes en cada topografía. Este método, permite cumplir con dos objetivos fundamentales: 1) Comparar registros por zona corporal a fin de detectar nuevas lesiones no existentes en controles anteriores y 2) comparar las imágenes dermatoscópicas de las lesiones registradas individualmente a fin de detectar cambios mínimos, ya que éstos, según sus características, pueden levantar sospecha de melanoma. De hecho, lesiones pigmentadas que no se muestran malignas con la dermatoscopía convencional, pueden transformarse en lesiones sospechosas de malignidad ante mínimos cambios dermatoscópicos registrados con estos métodos. Es por esto que el SDDMC está indicado formalmente en pacientes con riesgo o antecedentes de melanoma y ha demostrado utilidad en el diagnóstico precoz de segundos melano- mas, ya que éstos, se diagnostican in situ o con un índice de Breslow significativamente menor al del primer melanoma, en comparación con pacientes que no se encuentran bajo SDDMC. ${ }^{10,11,12}$

Dado que el antecedente personal de melanoma es el mayor factor de riesgo para presentar un segundo melanoma ${ }^{13} \mathrm{y}$ teniendo en cuenta que el tratamiento con vemurafenib se asocia con aparición de segundos melanomas per se, el grupo de pacientes con melanoma en tratamiento con vemurafenib tiene clara indicación de SDDMC.

Presentamos dos casos clínicos de pacientes con antecedentes de melanoma en plan de SDDMC.

Caso 1. Paciente de 52 años, melanoma diagnosticado hace siete años interescapular con ganglio centinela positivo y mestástasis cutáneas, en tratamiento con vemurafenib hace cuatro años en monoterapia a dosis de $960 \mathrm{mg}$ cada 12 horas vía oral. El paciente es controlado hasta el día de la fecha con SDDMC a corto plazo (cada 3 meses). En la evolución ha presentado cambios en sus nevos preexistentes (Figura 1) y aparición de nuevas lesiones pigmentadas de las cuales destacamos aparición de dos melanomas in situ (Figura2).

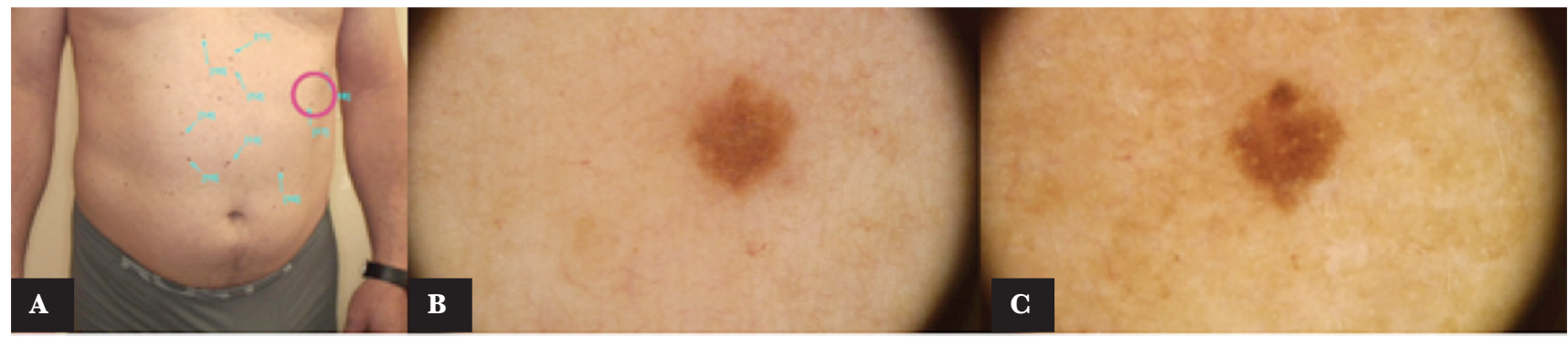

Figura 1

Imagen macroscópica A. Lesión pigmentada plana de $3 \mathrm{~mm}$ de eje mayor, a la dermatoscopía presenta patrón reticular B. bajo seguimiento digital en 3 meses agrega mancha periférica de pigmento en polo superior $\mathbf{C}$. Exéresis con estudio histopatológico que informó nevo atípico.

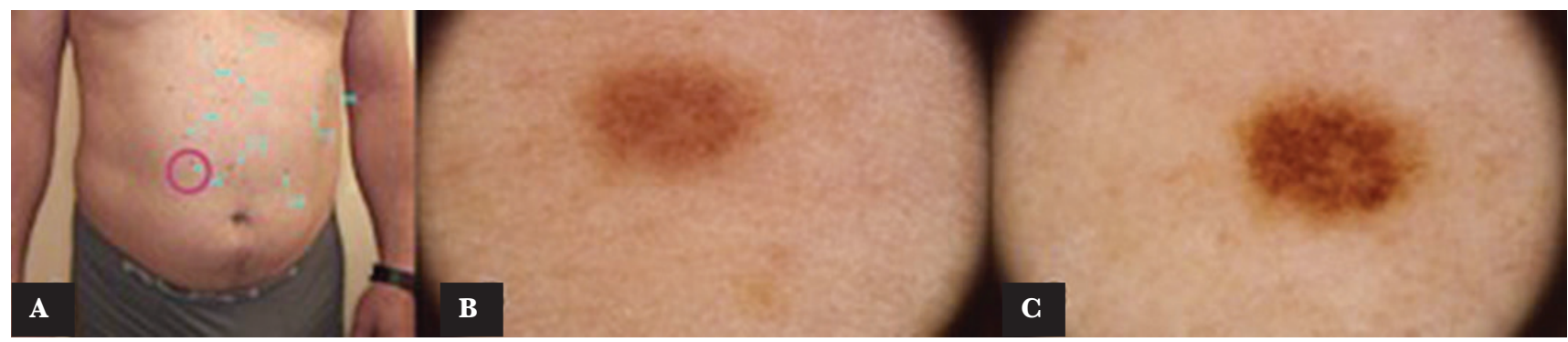

Figura 2

Lesión en abdomen imagen macroscópica A. lesión pigmentada plana dada por un patrón reticular B. cambios presentes durante el seguimiento digital a 3 meses, dados por refuerzo de la red pigmentada y aumento de la pigmentación global con aparición de glóbulos en periferia C. Exéresis con estudio histopatológico que informó melanoma. 
Tabla 1

Casos clínicos y hallazgos durante el seguimiento digital

\section{Tratamiento}

CASO 1 Vemurafenib $960 \mathrm{mg}$ cada $12 \mathrm{hs} \mathrm{v/o}$

\section{Hallazgos}

Agrega mancha periférica de pigmento en polo superior de nevo preexistente, aumento global de la pigmentación de los nevos, apari ción de nuevas lesiones pigmentadas y dos melanoma in-situ

Aumento global de la pigmentación de nevos, aparición de metástasis cutánea
Caso 2. Paciente de 53 años, melanoma metastásico diagnosticado hace 6 años en dorso

por lo cual inicia combinación de vemurafenib 960 mg vía oral cada 12 horas más cobimetinib $60 \mathrm{mg}$ vía oral por día en abril del corriente año, a los 15 días del inicio del tratamiento presenta rash maculo papular extenso por lo cual se disminuye la dosis a vemurafenib $480 \mathrm{mg}$ cada 12 horas y cobimetinib 60 mg día hasta el momento actual.

Se destaca durante el SDDMC al mes de tratamiento una nueva lesión que resultó ser una metástasis cutánea (Figura 3) y cambios dermatoscópicos significativos en nevos melanocíticos como aumento global de la pigmentación de los mismos (Figura 4).

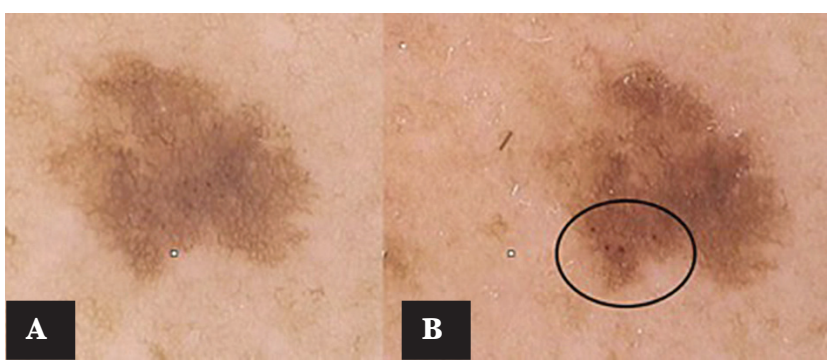

Figura 4

Nevo melanocítico en seguimiento digital desde hace 4 años estable A. A los 4 meses de Tratamiento con vemurafenib y cobimetinib muestra aparición de glóbulos en polo inferior B.cambios presentes en varias lesiones en la misma paciente.

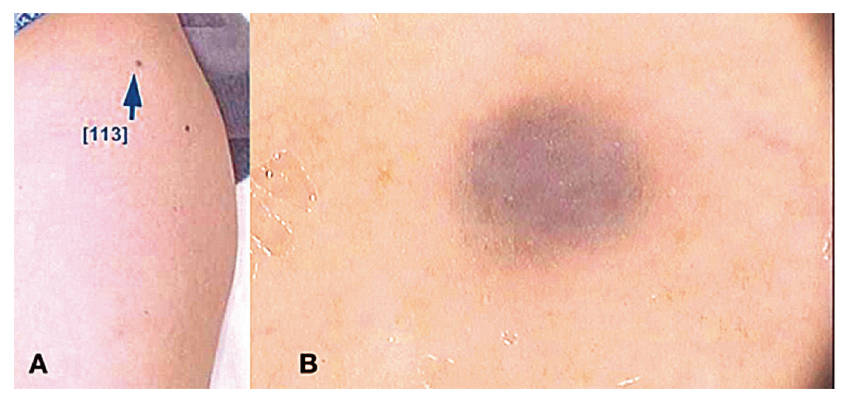

Figura 3

Lesión nueva detectada al mes de seguimiento digital dermatoscopico y mapeo corporal en muslo izquiedo. Exéresis con estudio histopatológico informó metástasis de melanoma.

\section{Discusión}

Dalle et al. fueron de los primeros en reportar aparición de segundos melanomas primarios en pacientes tratados con vemurafenib. ${ }^{12}$ Los autores estudiaron 120 pacientes con melanoma metastásico tratados con vemurafenib a dosis de $960 \mathrm{mg}$ dos veces al día mediante un examen clínico cutáneo mensual que incluyó dermatoscopía y seguimiento digital, logrando el diagnóstico de 25 segundos melanomas primarios en este grupo. ${ }^{14}$ Estos hallazgos fueron confirmados por varios grupos ${ }^{15,16}$ quienes reportan además, cambios en los nevos como aumento de pigmentación y aumento de lesiones melanocíticas en pacientes tratados con un inhibidor selectivo de BRAF.

En 2014, Perier-Muzet et al. publicaron un estudio longitudinal que incluyó 2155 lesiones melanocíticas de 42 pacientes en tratamiento con vemurafenib. Los cambios dermatoscópicos más frecuentemente reportados en nevos preexistentes fueron: cambio en la coloración global de la lesión $(15 \%)$ y la aparición de 
glóbulos (12\%). La mayoría de éstos se hallaron en los 5 primeros meses posteriores al inicio del tratamiento. Se diagnosticó un nuevo melanoma primario en 14 de $42(21 \%)$ pacientes. $^{5}$

No sólo se han reportado cambios con inhibidores de BRAF, también se han reportado varios casos de pacientes cuyos nevos cambiaron significativamente durante el tratamiento combinado con inhibidores de BRAF e inhibidores MEK, tal es el caso de la combinación dabrafenib más trametinib, en donde se observó aclaramiento o regresión de lesiones melanocíticas. ${ }^{17,18,19,20,21}$

Recientemente, Perier-Muzet et al. ${ }^{21}$ publicaron otro estudio basado en 13 pacientes en tratamiento con inhibidor de BRAF o dabrafenib con trametinib (D \& T) que documentó los cambios globales de pigmentación en 427 nevos.

De acuerdo a lo antedicho, son varios los estudios que sugieren que diferentes medicamentos sistémicos para el tratamiento de melanoma pueden tener efectos sobre la pigmentación de las lesiones melanocíticas $^{21,22,23,24}$, una posible explicación a esto sería la proliferación paradójica de los melanocitos BRAF de tipo salvaje cuando se exponen a un inhibidor de BRAF. Se ha propuesto que el inhibidor de BRAF actúa para inducir la dimerización, la localización de la membrana y la interacción con RAS-GTP en melanocitos BRAF de tipo salvaje, facilitando la activación de la vía RAS-RAF-MEK -ERK y aumentando así el crecimiento de los tipo melanocitos BRAF. ${ }^{23}$

Tanto en la serie de Dalle et al. ${ }^{14}$ como en la de Zimmer et al. ${ }^{15}$ la mayoría de los melanomas se desarrollaron a las pocas semanas de tratamiento y se detectaron en una etapa clínica temprana. Es por esto que Perier-Muzet et al. sugieren seguimiento digital mensual por al menos los primeros 3 meses de tratamiento seguido por uno cada 3 meses. ${ }^{5}$

Dado que bajo el tratamiento con vemurafenib, se pueden observar cambios significativos en los nevos y que ellos ocurren muy rápidamente luego del inicio de dicho fármaco, las lesiones deben ser valoradas minuciosamente y los criterios clínicos que usa el dermatólogo para determinar la exéresis de una lesión no son los mismos que en otros pacientes. La decisión debe basarse en la experiencia clínico dermatoscópica del especialista manteniendo un índice de sospecha mayor que en pacientes de otros grupos de alto riesgo (pacientes con predisposición genética al melanoma, pacientes con síndrome de nevos atípicos, o pacientes con antecedentes personales o familiares de múltiples melanomas). Los dos casos presentados ponen de manifiesto la utilidad del SDDMC seriado en estos pacientes, en ambos casos el diagnóstico de aparición de nuevas lesiones y la comparación dermatoscópica de las mismas permitió diagnóstico precoz de segundos melanomas que resultaron in situ y metástasis cutáneas resecables.

\section{Gonalusión}

Es indiscutible que el SDDMC es de gran ayuda en el seguimiento y valoración de los pacientes con riesgo de melanoma permitiendo un diagnóstico precoz de lesiones malignas y minimizando la exéresis innecesaria de lesiones benignas. Dado que el uso de terapias oncológicas dirigidas es cada vez más frecuente, el grupo de pacientes con melanoma metastásico en tratamiento con BRAFi y/o BRAFi y MEKi se encuentra en crecimiento. Esto redunda en un aumento de la sobrevida pero como contrapartida negativa expone a los pacientes a efectos adversos y al riesgo de aparición de segundos melanomas. En este sentido, el SDDMC se presenta como una herramienta fundamental ya que optimiza el diagnóstico de segundos melanomas e incluso metástasis cutáneas que suelen ser resecables. 


\section{REFERENCIAS}

1. Anforth RM, Carlos GR, Scolyer RA, Chou S, Fernandez-Peñas P. Eruptive naevi in a patient treated with LGX818 for BRAF mutant metastatic melanoma. Melanoma Res. 2015; 25(1): 91-4

2. Haenssle HA, Kraus SL, Brehmer F, Kretschmer L, Volker B, Asper $\mathrm{H}$, et al. Dynamic changes in nevi of a patient with melanoma treated with vemurafenib: importance of sequential dermoscopy. Arch Dermatol. 2012;148(10): 1183-5

3. Chu EY, Wanat KA, Miller CJ, Amaravadi RK, Fecher LA, Brose MS, et al. Diverse cutaneous side effects associated with BRAF inhibitor therapy: a clinicopathologic study. J Am Acad Dermatol. 2012; 67(6): 1265-72

4. Dalle S, Poulalhon N, Thomas L. Vemurafenib in melanoma with BRAF V600E mutation. N Engl J Med. 2011; 365(15): 1448-9; author reply 1450

5. Perier-Muzet M, Thomas L, Poulalhon N, Debarbieux S, Bringuier $\mathrm{PP}$, Duru G, et al. Melanoma patients under vemurafenib: prospective follow-up of melanocytic lesions by digital dermoscopy. J Invest Dermatol. 2014; 134(5): 1351-8

6. Boussemart L, Girault I, Malka-Mahieu H, Mateus C, Routier E, Rubington M, et al. Secondary tumors arising in patients undergoing BRAF inhibitor therapy. Cancer Res. 2016;76(6):1476-84

7. Sinha R, Edmonds K, Newton-Bishop JA, Gore ME, Larkin J, Fearfield L. Cutaneous adverse events associated with vemurafenib in patients with metastatic melanoma: practical advice on diagnosis, prevention and management of the main treatment-related skin toxicities. Br J Dermatol 2012; 167(5):987-94

8. Bafounta ML, Beauchet A, Aegerter P, Saiag P. Is dermoscopy (epiluminescence microscopy) useful for the diagnosis of melanoma? Results of a meta-analysis using techniques adapted to the evaluation of diagnostic tests. Arch Dermatol 2001; 137(10): 1343-1350

9. Mayer J. Systematic review of the diagnostic accuracy of dermatoscopy in detecting malignant melanoma. Med J Aust.1997; 18;167(4):206-10

10. Tromme I, Sacre L, Hammouch F, Legrand C, Marot L, Vereecken P, et al. Availability of digital dermoscopy in daily practice dramatically reduces the number of excised mela- nocytic lesions: results from an observational study. Br J Dermatol. 2012; 167(4): 778-86

11. Salerni G, Carrera C, Lovatto L, Puig-Butille JA, Dadenas C, Plana E, et al. Benefits of total body photography and digital dermatoscopy ("two-step method of digital follow-up") in the early diagnosis of melanoma in patients at high risk for melanoma. J Am Acad Dermatol. 2012 Jul;67(1):e17-27

12. Malvehy J., Puig S. Follow-up of melanocytic skin lesions with digital total-body photography and digital dermoscopy: a two-step method, Clin Dermatol.,2002, 20: 297-304

13. Siskind V, Hughes MC, Palmer JM, Symmons JM, Aitken JF, Martin NG, et al. Nevi, family history, and fair skin increase the risk of second primary melanoma. J Invest Dermatol. 2011; 131(2):461-7

14. Dalle S, Poulalhon N, Debarbieux S, Thomas L. Second primary melanomas on treatment with vemurafenib. Br J Dermatol. 2013; 168(4):887-8

15. Zimmer L, Hillen U, Livingstone E, Lacouture ME, Busman K, Carvajal RD, et al. Atypical melanocytic proliferations and new primary melanomas in patients with advanced melanoma undergoing selective BRAF inhibition. J Clin Oncol. 2012; 30(19):2375-83

16. Huang V, Hepper D, Anadkat M, Cornelius L. Cutaneous toxic effects associated with vemurafenib and inhibition of the BRAF pathway. Arch Dermatol. 2012; 148(5):628-38

17. Chen FW, Tseng D, Reddy S, Daud AI, Swetter SM. Involution of eruptive melanocytic nevi on combination BRAF and MEK inhibitor therapy. JAMA Dermatol. 2014; 150(11): 1209-12

18. Green JS, Norris DA, WisellJ. Novel cutaneous effects of combination chemotherapy with BRAF and MEK inhibitors: a report of two cases. Br J Dermatol. 2013; 169(1): 172-6

19. McClenahan P, Lin LL, Tan JM, Flewell-Smith R, Schaider H, Jagirdar $\mathrm{K}$ et al. BRAFV600E mutation status of involuting and stable nevi in dabrafenib therapy with or without trametinib. JAMA Dermatol. 2014; 150(10): 1079-82

20. Giurcaneanu C, Nitipir C, Popa LG, Forsea AM, Popescu I, Bumbacea RS. Evolution of melanocytic nevi under vemurafenib, followed by combination therapy with dabrafenib and trametinib for metastatic melanoma. Acta Dermatovenerol Croat. 2015; 23(2): $114-21$

21. Perier-Muzet M, Boespflug A, Poulalhon N, Caramel J, Breton AL, Thomas L, et al. Dermoscopic evaluation of melanocytic nevi changes with combined mitogen-activated protein kinase pathway inhibitors therapy for melanoma. JAMA Dermatol. 2016; 152(10): $1162-1164$

22. Zhao CY, Eun Hwang S, Wakade D, Carlos G, Anforth R, FernandezPenas P. Melanocytic lesion evolution patterns with targeted therapies and immunotherapies for advanced metastatic melanoma: An observational study. Australas J Dermatol. 2017;58(4):292-8

23. Hatzivassiliou G, Song K, Yen I, Brandhuber BJ, Anderson DJ, Alvarado R, et al. RAF inhibitors prime wild-type RAF to activate the MAPK pathway and enhance growth. Nature.2010;18;464(7287): $431-5$

24. Hwang SJ, Carlos G, Wakade D, Byth K, Kong BY, Chou S et al. Cutaneous adverse events (AEs) of anti-programmed cell death (PD)1 therapy in patients with metastatic melanoma: a single-institution cohort. J Am Acad Dermatol. 2016; 74(3): 455-61.el 